\title{
Location of the meso-pancreatoduodenum as a regional lymphatic basin for pancreatic head carcinoma
}

\author{
HIROFUMI TERAKAWA ${ }^{1}$, HIROHISA KITAGAWA ${ }^{1}$, ISAMU MAKINO ${ }^{1}$, HIRONORI HAYASHI ${ }^{1}$, \\ KATSUNOBU OYAMA $^{1}$, HISATOSHI NAKAGAWARA ${ }^{1}$, TOMOHARU MIYASHITA ${ }^{1}$, HIDEHIRO TAJIMA ${ }^{1}$, \\ HIROYUKI TAKAMURA ${ }^{1}$, SACHIO FUSHIDA ${ }^{1}$, NORIYUKI OZAKI ${ }^{2}$ and TETSUO OHTA ${ }^{1}$
}

Departments of ${ }^{1}$ Gastroenterologic Surgery and ${ }^{2}$ Functional Anatomy, Graduate School of Medical Science, Kanazawa University, Kanazawa 920-8641, Japan

Received December 26, 2015; Accepted February 27, 2017

DOI: $10.3892 / \mathrm{ol} .2017 .6138$

\begin{abstract}
The meso-pancreatoduodenum is the primary site of tumor infiltration in patients with pancreatic head cancer, with numerous patients exhibiting lymph node metastases. Effective dissection of the regional lymphatic basin requires knowledge of the patterns of the arterial branches. The present study examined the patterns of the arteries feeding the pancreatic head and the distribution of the meso-pancreatoduodenum. The present study included 123 patients with pancreatic cancer who underwent contrast-enhanced preoperative 64-multidetector-computed tomography to determine the routes of the inferior pancreaticoduodenal and first jejunal arteries. Surgical specimens and cadavers were also evaluated histologically to clarify the distribution of the meso-pancreatoduodenum. The feeding arteries were divided into three types, with $64.2 \%$ of patients having type $\mathrm{A}$, $28.4 \%$ having type $\mathrm{B}$ and $7.3 \%$ having type $\mathrm{C}$ branches. The branches emerged from the back or left side of the superior mesenteric artery and ran to the far side of the pancreatic head in an arc. Consequently, the meso-pancreatoduodenum had a roll-shaped appearance, surrounding the trunk arteries and extending to the left side of the superior mesenteric artery. Dissecting the right and left sides of the superior mesenteric artery during lymphadenectomy could improve the effectiveness of resection.
\end{abstract}

Correspondence to: Dr Hirofumi Terakawa, Department of Gastroenterologic Surgery, Graduate School of Medical Science, Kanazawa University, 13-1 Takara-machi, Kanazawa 920-8641, Japan

E-mail: hirofumi_terakawa@yahoo.co.jp

Key words: meso-pancreatoduodenum, regional lymphatic basin, pancreatic head carcinoma, lymphatic vessel involvement, 64-multidetector-computed tomography

\section{Introduction}

The prognosis of patients with pancreatic head cancer (PHC) is poor. Curative surgical resection is the main treatment modality contributing to good prognosis $(1,2)$. Even following curative pancreaticoduodenectomy, however, numerous patients experience local recurrence around the superior mesenteric artery (SMA) margin $(3,4)$, adversely affecting prognosis. Curative surgical resection requires control of tumor infiltration and lymph node metastasis. Standard lymphadenectomy instead of extended lymphadenectomy has been recommended as the cure for PHC (5). Lymphatic vessels form networks, allowing lymph node metastases to spread. Standard lymphadenectomy should therefore include identification of the primary site of lymph node metastasis and its regional lymphatic basin.

The mesopancreas, is defined as the soft connective tissue located between the SMA and the uncinate process, or the structure situated to the right side of the SMA, and is regarded as the primary site of cancer cell infiltration $(3,6,7)$. Excision of the entire mesopancreas can result in complete clearance of peripancreatic retroperitoneal tissue and improve the prognosis of patients with PHC. However, numerous patients with PHC possess lymph node metastases on the left side of the SMA $(8,9)$, and lymphadenectomy involving the left side of the SMA does not include the mesopancreas.

The meso-pancreatoduodenum (meso-pd), consisting of a cluster of soft connective tissue situated along the inferior pancreaticoduodenal artery (IPDA) and the first jejunal artery (FJA), is thought to be a site of lymphatic spread, and total excision of the meso-pancreatoduodenum (tMPDe) is regarded as necessary for pathological cure (10). Although tMPDe includes the left side of the SMA, arterial branches from the SMA to the head of the pancreas exhibit various patterns. The present study examined the patterns of the arteries feeding the pancreatic head and the distribution of the meso-pd.

\section{Patients and methods}

Between January 2003 and December 2013, 123 patients with pancreatic cancer underwent preoperative 64-multidetector-computed tomography (CT). Written informed consent was obtained from each patient for the use of their data 
in the present study. Iodinated contrast material $(350 \mathrm{mg} / \mathrm{ml})$ was delivered at a dose of $1.8 \mathrm{ml} / \mathrm{kg}$ over $30 \mathrm{sec}$. Early and late arterial phase images were captured at 25 and $40 \mathrm{sec}$, respectively. These images were examined to determine the routes of the IPDA and the FJA.

The branches of the pancreatic head were categorized into three types as described (11) (Fig. 1). In type A, the IPDA formed a common vessel with the FJA; in type B, the IPDA branched directly from the SMA; and in type $\mathrm{C}$, the anterior inferior pancreaticoduodenal artery and the posterior inferior pancreaticoduodenal artery (PIPDA) branched out separately from the SMA. The angles formed at the point of emergence from the SMA of these branches were measured using horizontal CT; using the SMA as the axis, the ventral side was considered $0^{\circ}$, the left side $90^{\circ}$ and the dorsal side $180^{\circ}$.

The surgical specimens and cadavers were assessed histologically to determine the distribution of meso-pd. The cadaveric study was conducted at the Department of Functional Anatomy, Kanazawa University (Kanazawa, Japan). All cadavers were donated to the university for medical education and study purposes. Cadavers with any intra-abdominal injury or gross intra-abdominal pathology were excluded. Dissection was started with a midline vertical abdominal incision. All the tissues between the pancreas and aorta, including the SMA, inferior vena cava, superior mesenteric vein and posterior abdominal wall, were preserved. The aorta and inferior vena cava were ligated and divided from the level of the celiac trunk to the level of the inferior mesenteric artery. The SMA was preserved at the level of the third duodenal portion. Surgical and cadaveric specimens were fixed in $10 \%$ formalin, cut horizontally into 5-mm tissue blocks corresponding with the CT scan images, dehydrated and embedded in paraffin. Sections $5 \mu \mathrm{m}$ in thickness were cut and stained with hematoxylin and eosin, elastica van Gieson and D2-40, the latter for detection of lymphatic vessels.

Immunohistochemical assays were performed on formalin-fixed paraffin-embedded tissues. Sections were cut $5 \mu \mathrm{m}$ thick, deparaffinized in xylene, rehydrated in graded alcohols (100\%) and washed in PBS. Slides were boiled in citrate buffer (pH 6.0) at $120^{\circ} \mathrm{C}$ for $5 \mathrm{~min}$ and allowed to cool for $20 \mathrm{~min}$. Endogenous peroxidases were blocked by incubation in $0.3 \%$ hydrogen peroxide in distilled water, followed by washing in PBS three times. Sections were incubated with D2-40 monoclonal antibody (cat. no. M3619; dilution, 1:50, Dako; Agilent Technologies, Inc., Santa Clara, CA, USA) for $90 \mathrm{~min}$ at room temperature, with immunohistochemical staining performed using an EnVision + horseradish peroxidase DAB system (Dako; Agilent Technologies, Inc.). The tissue sections were subsequently counterstained with hematoxylin and dehydrated through a graded ethanol series (100\%) and xylene three times.

\section{Results}

The characteristics of patients enrolled in this study are summarized in Table I. All three types of branching of the IPDA and FJA in the pancreatic head were observed in our patients, with $79(64.2 \%)$ having type A, 35 (28.5\%) having type B and 9 (7.3\%) having type C branches (Table II). In type A, the vessel common to the IPDA and FJA emerged
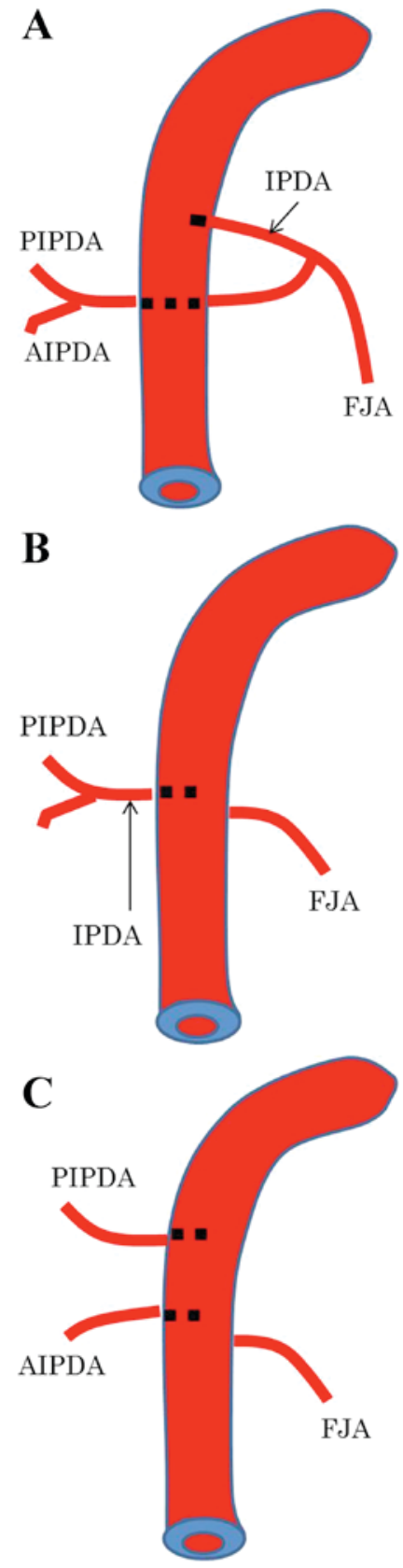

Figure 1. Illustration of the three types of IPDA branching. (A) Type A: The IPDA forms a common vessel with the FJA. (B) Type B: The IPDA branches directly from the SMA. (C) Type C: The AIPDA and PIPDA branch from separate parts of the SMA. IPDA, inferior pancreaticoduodenal artery; FJA, first jejunal artery; SMA, superior mesenteric artery; AIPDA, anterior inferior pancreaticoduodenal artery; PIPDA, posterior inferior pancreaticoduodenal artery.

between the left and dorsal sides of the SMA $\left(148 \pm 40.0^{\circ}\right)$. In types $\mathrm{B}$ and $\mathrm{C}$, the IPDA and PIPDA emerged from the dorsal side of the SMA, at $187 \pm 47.0^{\circ}$ and $182 \pm 37.4^{\circ}$, respectively (Fig. 2; Table II). The routes of the IPDA, IPDA/FJA common vessel and PIPDA exhibited bending.

The distribution of the meso-pd was determined by evaluating the histological continuity of tissue in surgical specimens and cadavers. Immunohistochemistry with antibodies to D2-40 revealed lymphatic vessels beside the collagen fibers in this area (Fig. 3). Collagen fibers, forming two fibrous bunches, were observed regularly in the soft tissue. Around the SMA, 
Table I. Location of pancreatic cancers by age and sex.

\begin{tabular}{lcc}
\hline $\begin{array}{l}\text { Location of the } \\
\text { pancreatic cancer }\end{array}$ & $\begin{array}{c}\text { Pancreatic } \\
\text { head }\end{array}$ & $\begin{array}{c}\text { Pancreatic body } \\
\text { and tail }\end{array}$ \\
\hline $\begin{array}{l}\text { Sex, male/female } \\
\text { Age, years, mean (range) }\end{array}$ & $63.5(34-81)$ & $66.5(45-84)$ \\
\hline
\end{tabular}

Table II. IPDA branching types of pancreatic cancers.

\begin{tabular}{lcc}
\hline $\begin{array}{l}\text { IPDA branching } \\
\text { type }\end{array}$ & $\begin{array}{c}\text { Number of patients, } \\
\mathrm{n}(\%)\end{array}$ & $\begin{array}{c}\text { Angle formed by } \\
\text { the IPDA or PIPDA }\end{array}$ \\
\hline Type A & $79(64.2)$ & $148 \pm 40.0^{\circ}$ \\
Type B & $35(28.4)$ & $187 \pm 47.0^{\circ}$ \\
Type C & $9(7.3)$ & $182 \pm 37.4^{\circ}$ \\
\hline
\end{tabular}

IPDA, inferior pancreaticoduodenal artery; PIPDA, posterior inferior pancreaticoduodenal artery. Type A, Type B and Type C. Type A: The IPDA forms a common vessel with the FJA. Type B: The IPDA branches directly from the SMA. Type C: The AIPDA and PIPDA branch from separate parts of the SMA.
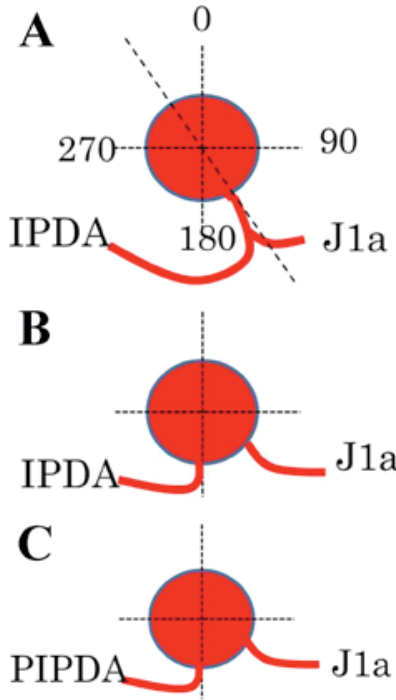

Figure 2. Illustration of the branching patterns of the IPDA, FJA, and PIPDA (A) In type A branching, the IPDA/FJA common vessel arises between the left and dorsal sides of the SMA. (B) In type B branching, the IPDA arises from the dorsal side of the SMA. (C) In type $C$ branching, the PIPDA arises from the dorsal side of the SMA. IPDA, inferior pancreaticoduodenal artery; FJA, first jejunal artery; PIPDA, posterior inferior pancreaticoduodenal artery; SMA, superior mesenteric artery.
A

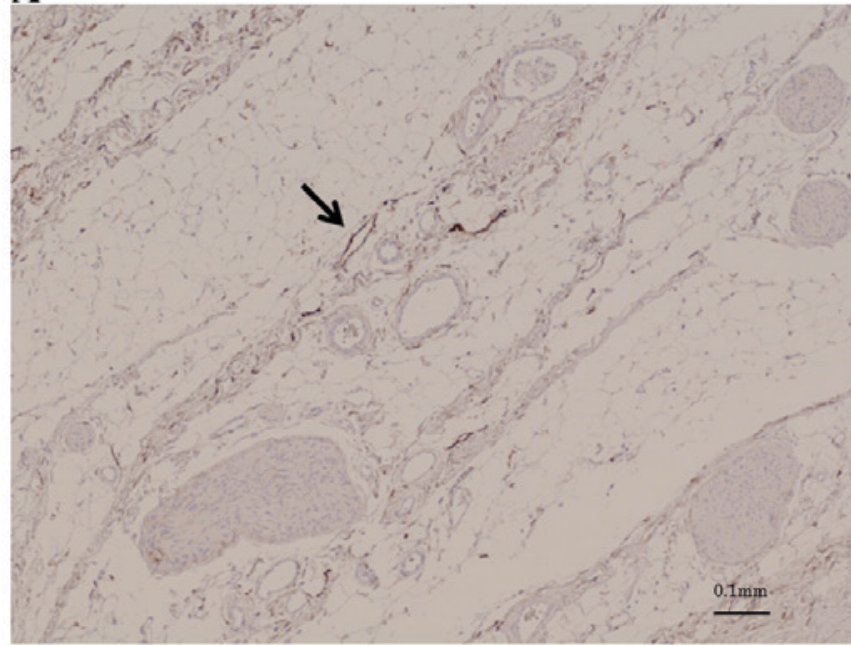

B

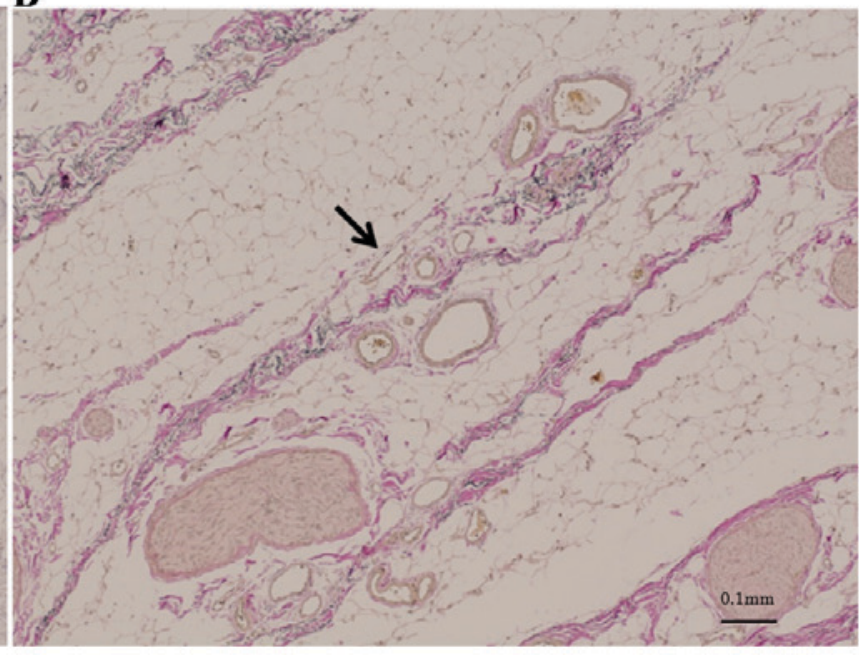

Figure 3. Patterns of D2-40 staining demonstrating (A) a lymph duct (arrow) adjacent to (B) collagen fibers. Magnification, x40.

which was covered grossly by fibrous tissue, collagen fibers formed concentric circles $\sim 3 \mathrm{~mm}$ thick, a structure called the superior mesenteric arterial plexus (PLsma) (12). By contrast, collagen fibers near the uncinated process ran vertically from the uncinated process of the pancreas to the PLsma, with continuity through the posterior and left side of the SMA (Fig. 4). These collagen fibers, which were also bent, gradually fused and joined with the PLsma, making the PLsma thicker as it proceeded towards the origin of the SMA (Fig. 5).

\section{Discussion}

We previously determined that the manner of lymphatic extension and nerve plexus infiltration of the PHC were dependent on whether the tumor originated from the embryonic dorsal or ventral pancreatic bud $(12,13)$. Tumors confined to the ventral pancreas extend toward the SMA, whereas tumors confined to the dorsal pancreas extend towards the common HA or hepatoduodenal ligament. If the tumor infiltrates deeply into the two areas, the cancer is likely to extend in the two directions. Therefore, the SMA margin is important for the carcinoma of the ventral pancreas.

The present study examined the distribution of the meso-pd, which was considered to be the mesentery of the embryonic ventral pancreas. The SMA margin is the most frequent site of $\mathrm{PHC}$ recurrence (3), despite the soft tissue on the right side of the SMA being regularly resected by pancreaticoduodenectomy. Lymphatic vessel involvement 
A

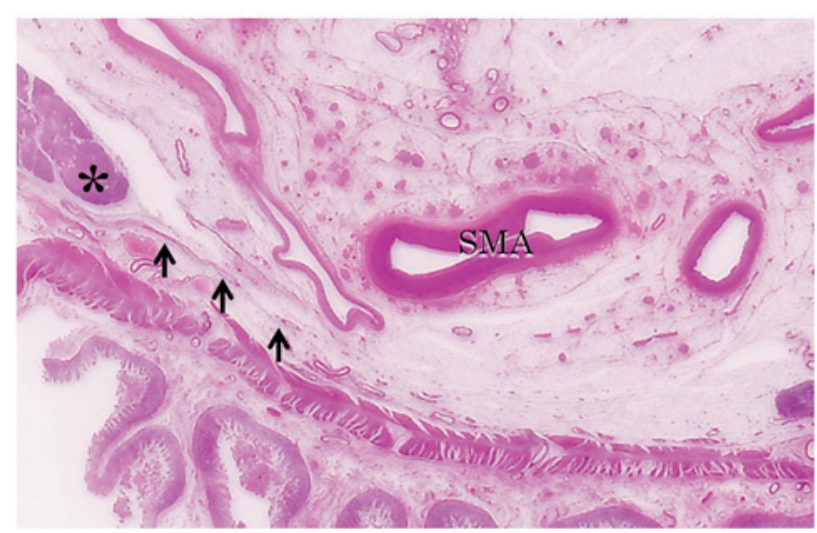

C

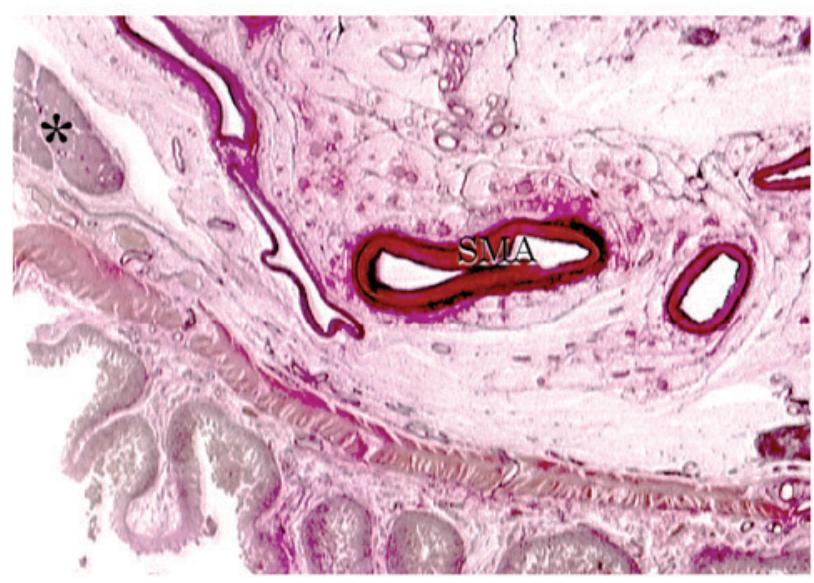

B

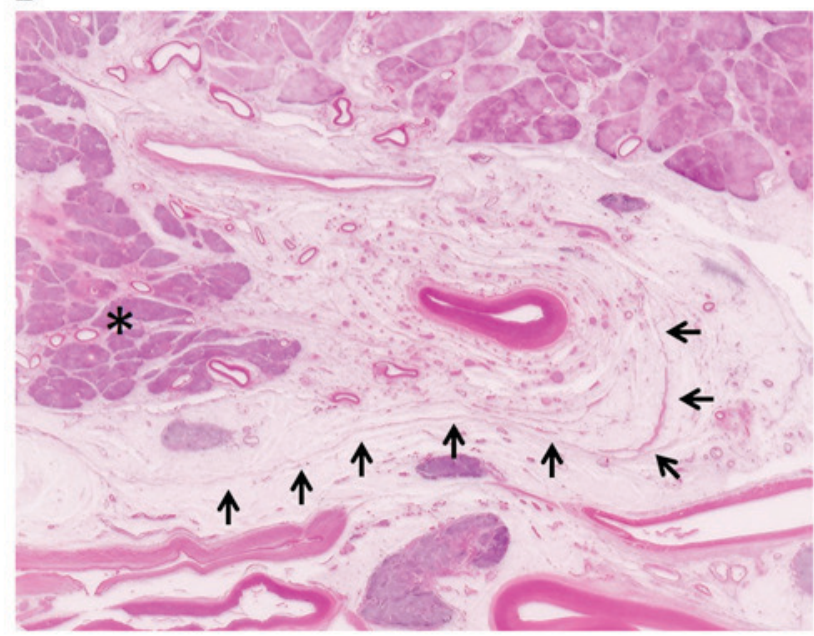

D

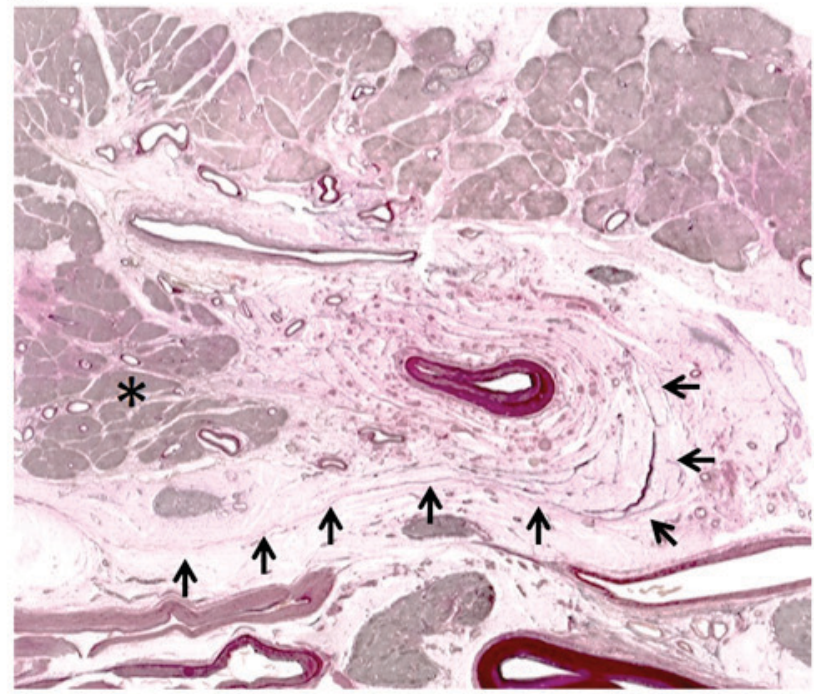

Figure 4. Histological assessment of collagen fibers in tissue sections. Hematoxylin and eosin staining revealed that (A) at the level of the third portion of the duodenum, the collagen fibers (arrows) that surround the SMA are not continuous with those surrounding the pancreatic head (asterisk) but gradually develop continuity at the (B) upper level. (C and D) Elastica van Gieson stained tissue sections. SMA, superior mesenteric artery.

is common in PHC. Regional lymphatic basin resection is required to avoid local recurrence, since this area is thought to remain the regional lymphatic basin of PHC subsequent to soft tissue resection of the right side of the SMA, resulting in local recurrence. The mesopancreas $(3,6,7)$, defined as the structure located on the right side of the SMA, is the primary site of cancer cell infiltration. However, lymph node metastasis is often observed on the left side of the SMA in patients with PHC $(8,9)$. Resection based on the mesopancreas may therefore be insufficient for curative resection of the regional lymphatic basin.

The meso-pd, consisting of a cluster of soft connective tissue situated along the IPDA and the FJA, is regarded as the site of lymphatic spread, with tMPDe regarded as necessary for pathological cure (10). Patterns of arterial branches differ in the pancreatic head. Dissection of the lymphatic basin in PHC patients requires assessment of these patterns by multi detector CT, thus clarifying the direction of the SMA. At emergence, the arteries feeding the pancreatic head can be classified into three types (11). All of these branches emerged from the back or left side of the SMA and ran to the far side of the pancreatic head in an arc. Therefore, the meso-pd was located in a roll shape around the trunk arteries, extending to the left side of the SMA.

By investigating the continuity of the meso-pd histologically in this area of soft tissue, it was revealed by immunohistochemistry that the lymphatic vessels were alongside the collagen fibers. The present study therefore examined the distribution of the meso-pd relative to the continuity of the collagen fibers. The meso-pd originates from the uncinated process of the pancreas and connects to the PLsma, defined as the left back side of the soft tissue around the SMA (14). The distribution of the meso-pd is the same as the route of the IPDA. Its lower limit was vertically above the third duodenal portion. This soft tissue is also the mesentery of the jejunum, which is the route of the lymphatics and the nerves connecting the jejunum. These findings suggest that the regional lymphatic basin of the pancreatic head spreads towards the left side of the SMA, with an appearance resembling a Swiss roll. This appearance was likely caused by intestinal rotation in the fetus 
A

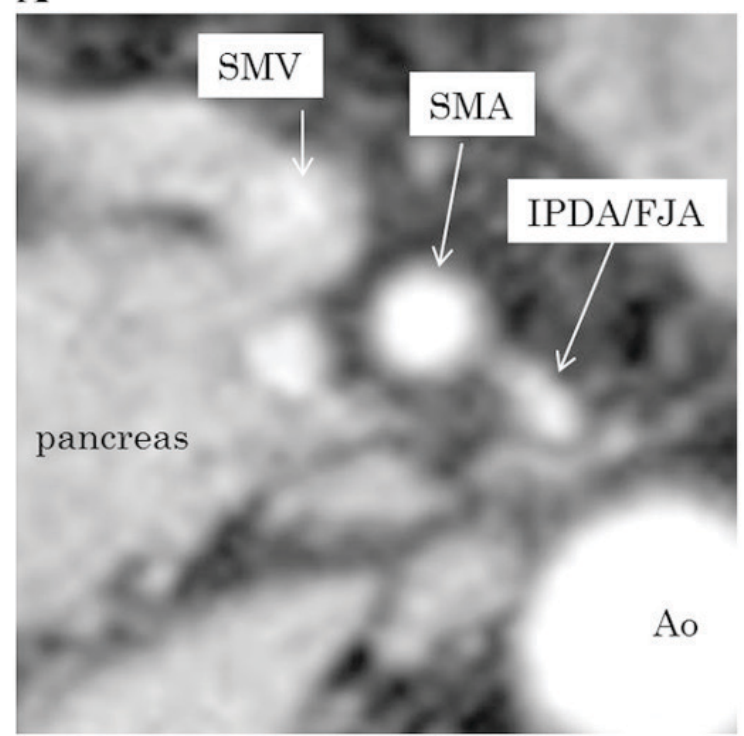

C

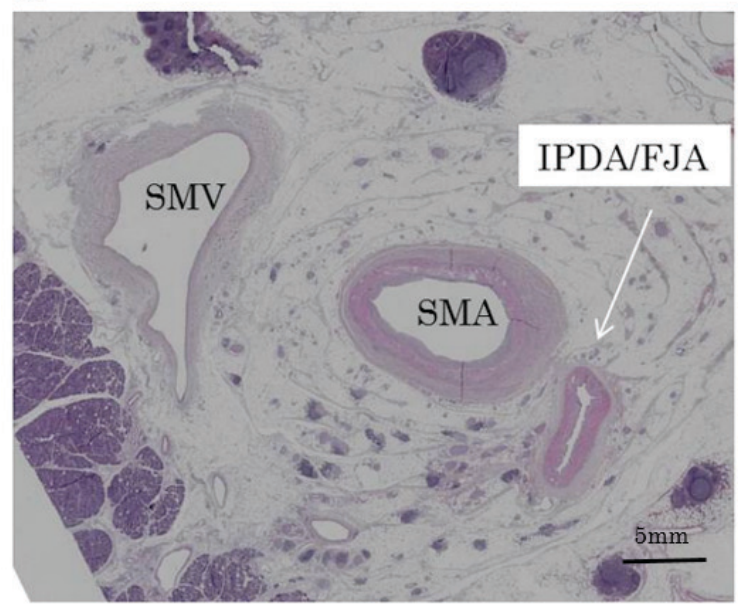

B

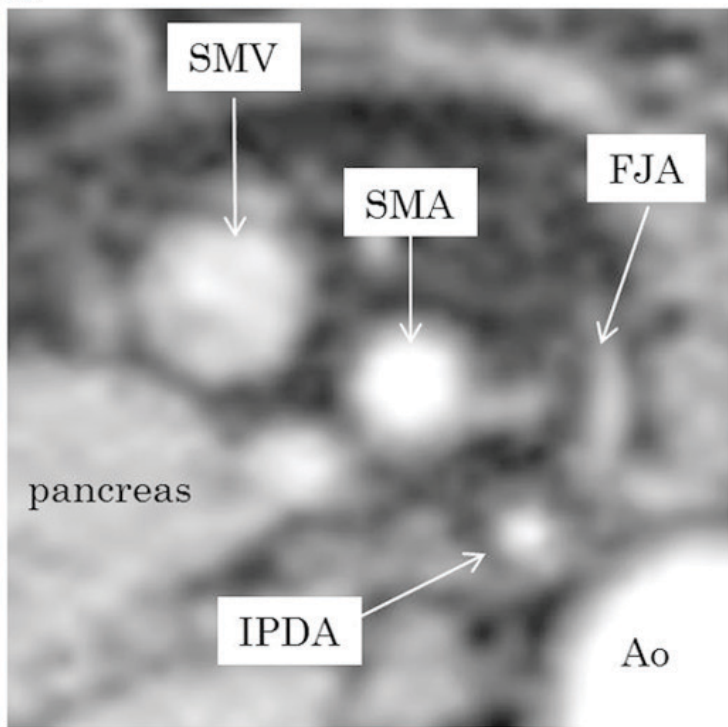

D

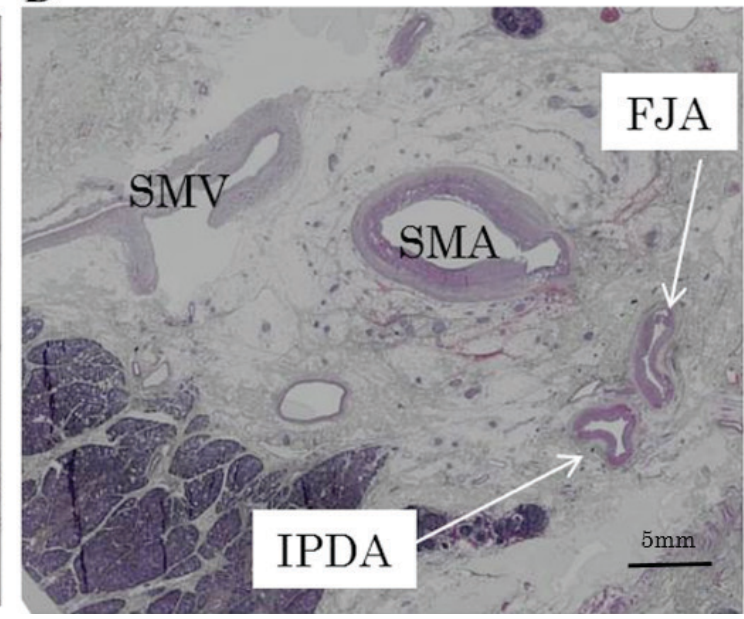

Figure 5. (A) CT scan showing the IPDA/FJA common vessel emerging between the left and dorsal sides of the SMA. (B) CT scan showing the IPDA branching from the FJA. (C) Surgical specimen showing the IPDA/FJA common vessel emerging between the left and dorsal sides of the SMA. (D) Surgical specimen showing the IPDA branching from the FJA. Magnification, x1. CT, computed tomography; IPDA, inferior pancreaticoduodenal artery; FJA, first jejunal artery; SMA, superior mesenteric artery; SMV, superior mesenteric vein; Ao, aorta.

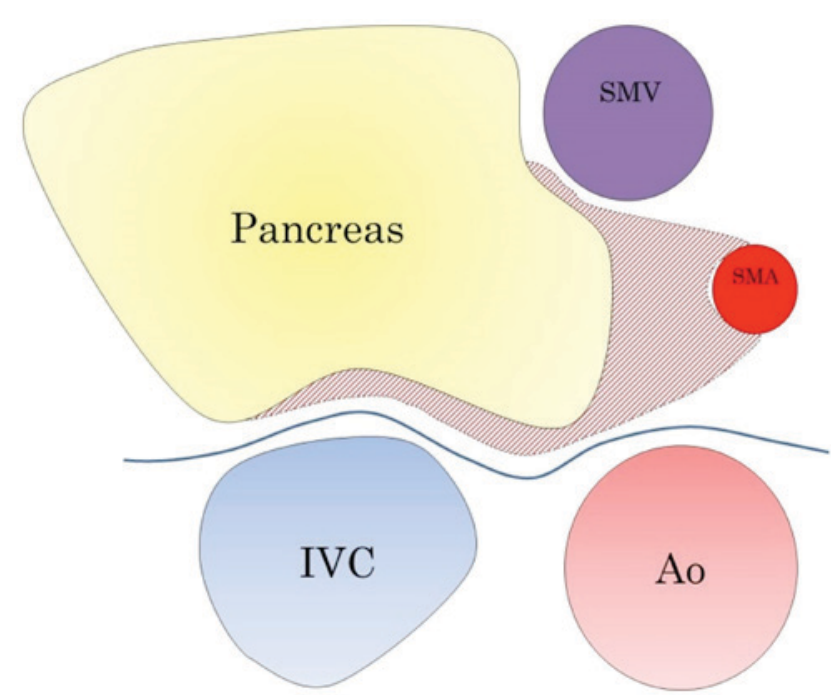

Figure 6. Diagram showing the mesopancreas situated to the right of the SMA (shaded area). SMA, superior mesenteric artery; SMV, superior mesenteric vein; Ao, aorta; IVC, inferior vena cava.
(Figs. 6 and 7) (15). The mid-gut rotated $270^{\circ}$ anticlockwise around the SMA, which acted as the axis. This resulted in a bend in the meso-pd, which subsequently extended to the left of the SMA.

The artery first approached during pancreatoduodenectomy has been revealed to contribute to the determination of resectability and reduction of bleeding; several procedures have been described (16). Early shutdown of arterial blood feeding the head of the pancreas is therefore important. The present evaluation of the location of the branches of the pancreatic head from the SMA supports the artery first approach. Among the various surgical methods, the mesenteric approach is considered optimal for en bloc resection since the meso-pd, which contains the nerve plexus and lymphatics associated with the head of the pancreas, extends from the right to the back side of the SMA. Thus, tMPDe has theoretical advantages for en bloc and curative resection of carcinomas of the ventral pancreas. By contrast, the mesentery corresponding to the embryonic dorsal pancreas is currently unclear, although it has been associated with the HA. Survival rate following HA 
A

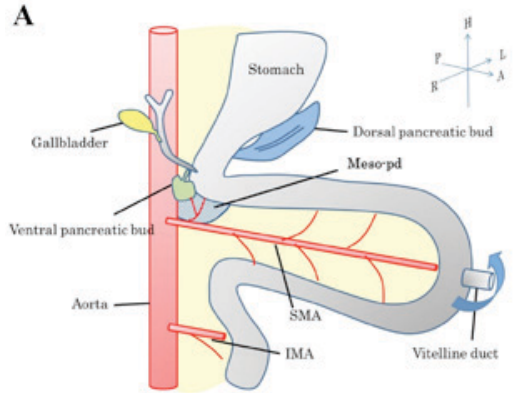

$\mathrm{C}$

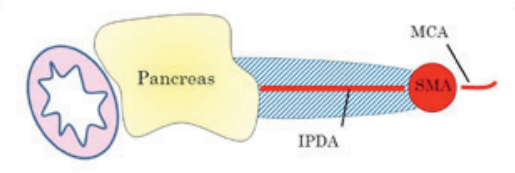

$\mathbf{E}$

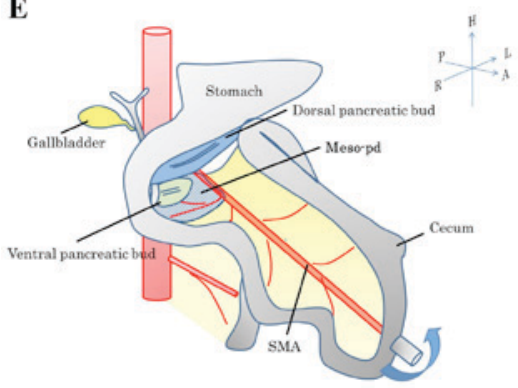

G

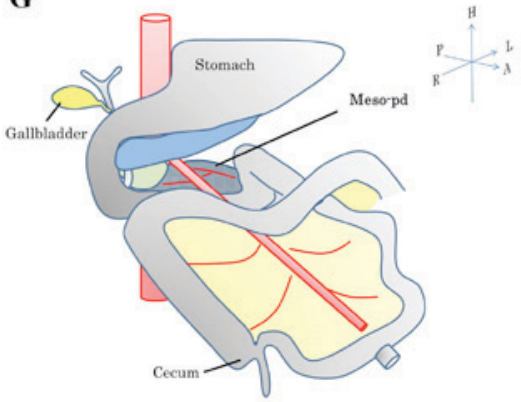

B

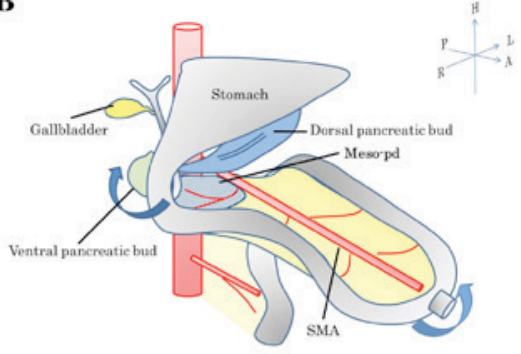

D

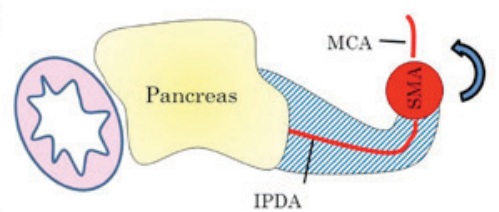

$\mathbf{F}$

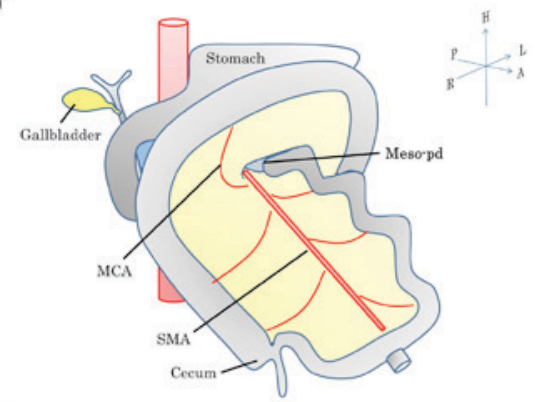

H

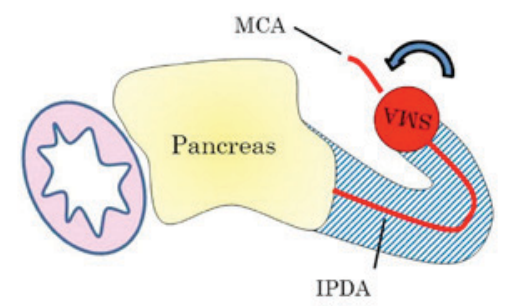

Figure 7. Diagrams showing (A) the position of the mid-gut prior to rotation during the prenatal period. (B) The position of the mid-gut following an anticlockwise rotation of $90^{\circ}$ around the SMA as the axis. (C) Horizontal slice of B. The meso-pd is linear. (E) Position of the mid-gut following anticlockwise rotation for another $90^{\circ}$. (D) Horizontal slice of c-1. A bend occurs in the meso-pd. (F) Position of the mid-gut following an anticlockwise rotation for another $90^{\circ}$; such a rotation is noted perinatally. (G) The transverse colon is pulled down until the third portion of the duodenum is contained within the meso-pd. (H) A bend recurring in the meso-pd, extending to the left of the SMA. SMA, superior mesenteric artery; IMA, inferior mesenteric artery; IPDA, inferior pancreaticoduodenal artery; MCA, middle colic artery; FJA, first jejunal artery.

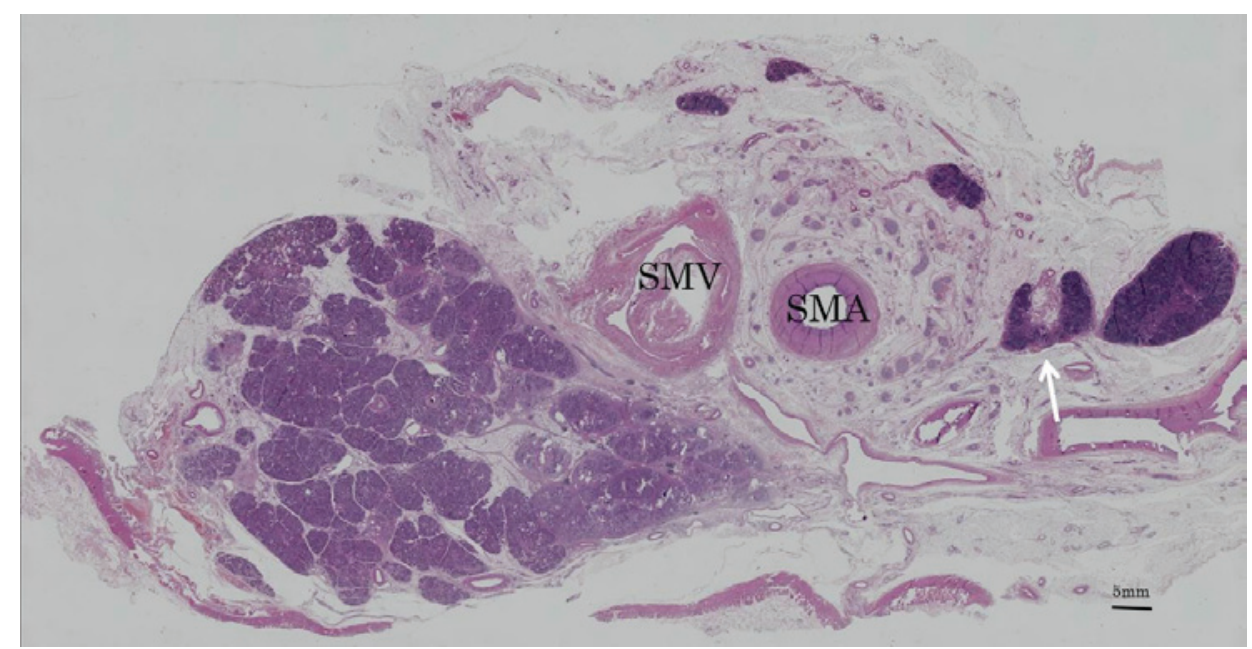

Figure 8. Photomicrograph of stained tissue section showing lymph node metastasis (white arrow) on the left side of the SMA. SMA, superior mesenteric artery; SMV, superior mesenteric vein. 
resection was poor in the present study and procedure, which focuses on the meso-pd, and was shown to be insufficient for treatment of carcinomas of the dorsal pancreas (17).

The present study concluded that the meso-pd, which spans the dorsal and left sides of the SMA (Fig. 8), is the proper mesentery of the pancreas and duodenum. Therefore, in patients with PHC, dissecting the right and left sides of the SMA during lymphadenectomy may be advantageous.

\section{References}

1. Wagner M, Redaelli C, Lietz M, Seiler CA, Friess H and Büchler MW: Curative resection is the single most important factor determining outcome in patients with pancreatic adenocarcinoma. Br J Surg 91: 586-594, 2004.

2. Stojadinovic A, Brooks A, Hoos A, Jaques DP, Conlon KC and Brennan MF: An evidence-based approach to the surgical management of resectable pancreatic adenocarcinoma. J Am Coll Surg 196: 954-964, 2003.

3. Gaedcke J, Gunawan B, Grade M, Szöke R, Liersch T, Becker H and Ghadimi BM: The mesopancreas is the primary site for R1 resection in pancreatic head cancer: Relevance for clinical trials. Langenbecks Arch Surg 395: 451-458, 2010.

4. Heye T, Zausig N, Klauss M, Singer R, Werner J, Richter GM, Kauczor HU and Grenacher L: CT diagnosis of recurrence after pancreatic cancer: Is there a pattern? World J Gastroenterol 17: 1126-1134, 2011.

5. Pedrazzoli S, DiCarlo V, Dionigi R, Mosca F, Pederzoli P, Pasquali C, Klöppel G, Dhaene K and Michelassi F: Standard versus extended lymphadenectomy associated with pancreaticoduodenectomy in the surgical treatment of adenocarcinoma of the head of the pancreas: A multicenter, prospective, randomized study. Lymphadenectomy Study Group. Ann Surg 228: 508-517, 1998

6. Gockel I, Domeyer M, Wolloscheck T, Konerding MA and Junginger T: Resection of the mesopancreas (RMP): A new surgical classification of a known anatomical space. World J Surg Oncol 5: 44, 2007

7. Adham M and Singhirunnusorn J: Surgical technique and results of total mesopancreas excision (TMpE) in pancreatic tumors Eur J Surg Oncol 38: 340-345, 2012.

8. Kayahara M, Nagakawa T, Ueno K, Ohta T, Tsukioka Y and Miyazaki I: Surgical strategy for carcinoma of the pancreas head area based on clinicopathologic analysis of nodal involvement and plexus invasion. Surgery 117: 616-623, 1995.
9. Noto M, Miwa K, Kitagawa H, Kayahara M, Takamura H, Shimizu K and Ohta T: Pancreas head carcinoma: Frequency of invasion to soft tissue adherent to the superior mesenteric artery. Am J Surg Pathol 29: 1056-1061, 2005.

10. Kawabata Y, Tanaka T, Nishi T, Monma H, Yano S and Tajima Y. Appraisal of a total meso-pancreatoduodenum excision with pancreaticoduodenectomy for pancreatic head carcinoma. Eur J Surg Oncol 38: 574-579, 2012.

11. Horiguchi A, Ishihara S, Ito M, Asano Y, Yamamoto T and Miyakawa S: Three-dimensional models of arteries constructed using multidetector-row CT images to perform pancreatoduodenectomy safely following dissection of the inferior pancreaticoduodenal artery. J Hepatobiliary Pancreat Sci 17: 523-526, 2010.

12. Kitagawa $H$, Ohta $T$, Makino I, Tani $T$, Tajima $H$, Nakagawara $H$, Ohnishi I, Takamura H, Kayahara M, Watanabe $\mathrm{H}$, et al: Carcinomas of the ventral and dorsal pancreas exhibit different patterns of lymphatic spread. Front Biosci 13: 2728-2735, 2008.

13. Makino I, Kitagawa H, Ohta T, Nakagawara H, Tajima $\mathrm{H}$, Ohnishi I, Takamura H, Tani T and Kayahara M: Nerve plexus invasion in pancreatic cancer: Spread patterns on histopathologic and embryological analyses. Pancreas 37: 358-365, 2008.

14. Japan Pancreas Society (ed): Classification of Pancreatic Carcinoma. 3rd English ed.: Kanehara \& Co., Ltd. Tokyo, 2011.

15. Shinohara H, Kurahashi Y, Kanaya S, Haruta S, Ueno M Udagawa $\mathrm{H}$ and Sakai Y: Topographic anatomy and laparoscopic technique for dissection of no. 6 infrapyloric lymph nodes in gastric cancer surgery. Gastric Cancer 16: 615-620, 2013.

16. Inoue Y, Saiura A, Yoshioka R, Ono Y, Takahashi M, Arita J, Takahashi Y and Koga R: Pancreatoduodenectomy with systematic mesopancreas dissection using a supracolic anterior artery-first approach. Ann Surg 262: 1092-1101. 2015.

17. Kitagawa H, Tajima H, Nakagawara H, Makino I, Miyashita T, Shoji M, Nakanuma S, Hayashi N, Takamura H, Ohta T and Ohtake H: En bloc vascular resection for the treatment of borderline resectable pancreatic head carcinoma. Mol Clin Oncol 2. 369-374, 2014. 